\title{
SINGULAR YAMABE METRICS BY EQUIVARIANT REDUCTION
}

\author{
ALI HYDER, ANGELA PISTOIA, AND YANNICK SIRE
}

\begin{abstract}
We construct singular solutions to the Yamabe equation using a reduction of the problem in an equivariant setting. This provides a non-trivial geometric example for which the analysis is simpler than in Mazzeo-Pacard program. Our construction provides also a non-trivial example of a weak solution to the Yamabe problem involving an equation with (smooth) coefficients.
\end{abstract}

\section{Contents}

1. Introduction 1

2. Preliminaries 3

2.1. Function spaces 3

2.2. The singular solution 4

2.3. The linearized operator around the singular solution 4

3. A scheme of the proof 5

4. The linear operator $L_{\varepsilon} \quad 6$

4.1. Injectivity of $L_{\varepsilon}$ on $C_{\mu, \mathcal{D}}^{2, \alpha}(\Omega \backslash \Sigma) \quad 6$

4.2. Uniform surjectivity of $L_{\varepsilon}$ on $C_{\mu, \mathcal{D}}^{2, \alpha}(\Omega \backslash \Sigma) \quad 9$

5. The non-linear term $Q \quad 12$

6. Appendix 14

References 15

\section{INTRODUCTION}

We consider the semilinear elliptic equation

$$
-\Delta_{\mathfrak{g}} u+h u=u^{p}, u>0, \text { on }(\mathcal{M}, \mathfrak{g})
$$

where $(\mathcal{M}, \mathfrak{g})$ is a $n$-dimensional compact Riemannian manifold without boundary, $h$ is a $C^{1}$-real function on $\mathcal{M}$ s.t. $-\Delta_{\mathfrak{g}}+h$ is coercive and $p>1$.

We are interested in finding solutions which are singular at $k$-dimensional manifolds for some integer $k \geq 1$.

In the critical case, i.e. $p=2_{n}^{*}-1:=\frac{n+2}{n-2}$ when the equation (1) coincides with the Yamabe equation (for $h=R_{\mathfrak{g}}$ the scalar curvature of $\mathcal{M}$ ), solutions singular at isolated points and at $k$-dimensional manifolds are known provided $k<(n-2) / 2$ (see [MP99, MS91, MP96, Sch88]).

In the present work, we provide a non trivial example of a geometric singular solution, in a much simpler setting than the original construction in [MP96]. Our idea is to rely on an equivariant reduction of the problem like the ones described for instance in [CP16].

Date: May 20, 2020.

2010 Mathematics Subject Classification. Primary: 35J60, Secondary: 35C20, 58J60.

Key words and phrases. Singular solution, Yamabe problem, warped product manifold, equivariant solution.

A. Hyder was supported by SNSF Grant No. P400P2-183866. A. Pistoia was partially supported by project Vain-Hopes within the program VALERE: VAnviteLli pEr la RicErca. 
For any integer $0 \leq k \leq n-3$ let $2_{n, k}^{*}=\frac{2(n-k)}{n-k-2}$ be the $(k+1)-$ st critical exponent. We remark that $2_{n, k}^{*}=2_{n-k, 0}^{*}$ is nothing but the critical exponent for the Sobolev embedding $\mathrm{H}_{\mathfrak{g}}^{1}(\mathcal{M}) \hookrightarrow \mathrm{L}_{\mathfrak{g}}^{q}(\mathcal{M})$, when $(\mathcal{M}, \mathfrak{g})$ is a $(n-k)$-dimensional Riemannian manifold. In particular, $2_{n, 0}^{*}=\frac{2 n}{n-2}$ is the usual Sobolev critical exponent.

In order to reduce the problem, we will consider the background manifold $\mathcal{M}$ to be given by a warped product. Let $(M, g)$ and $(K, \kappa)$ be two riemannian manifolds of dimensions $N$ and $k$, respectively. Let $\omega \in C^{2}(M), \omega>0$ be a given function. The warped product $\mathcal{M}=M \times{ }_{\omega} K$ is the product (differentiable) $n$-dimensional $(n=N+k)$ manifold $M \times K$ endowed with the riemannian metric $\mathfrak{g}=g+\omega^{2} \kappa$. The function $\omega$ is called the warping function. For example, every surface of revolution (not crossing the axis of revolution) is isometric to a warped product, with $M$ the generating curve, $K=S^{1}$ and $\omega(x)$ the distance from $x \in M$ to the axis of revolution.

It is not difficult to check that if $u \in C^{2}\left(M \times{ }_{\omega} K\right)$ then

$$
\Delta_{\mathfrak{g}} u=\Delta_{g} u+\frac{m}{\omega} g\left(\nabla_{g} u \nabla_{g} u\right)+\frac{1}{\omega^{2}} \Delta_{\kappa} u .
$$

Assume $h$ is invariant with respect to $K$, i.e. $h(x, y)=h(x)$ for any $(x, y) \in M \times K$. If we look for solutions to (1) which are invariant with respect to $K$, i.e. $u(x, y)=v(x)$ then by (2) we immediately deduce that $u$ solves (1) if and only if $v$ solves

$$
-\Delta_{g} v-\frac{m}{\omega} g\left(\nabla_{g} v, \nabla_{g} v\right)+h v=v^{p} \quad \text { in }(M, g) \text {. }
$$

or equivalently

$$
-\operatorname{div}_{g}\left(\omega^{N} \nabla_{g} v\right)+\omega^{N} h v=\omega^{N} v^{p}, v>0 \quad \text { in }(M, g) .
$$

It is clear that if $v$ is a solution to problem (3) which is singular at a point $\xi_{0} \in M$ then $u(x, y)=v(x)$ is a solution to problem (1) which is singular only on the fiber $\left\{\xi_{0}\right\} \times K$, which is a $k$-dimensional submanifold of $M \times_{\omega} K$. It is important to notice that the fiber $\left\{\xi_{0}\right\} \times K$ is totally geodesic in $M \times_{\omega} K$ (and in particular a minimal submanifold of $M \times_{\omega} K$ ) if $\xi_{0}$ is a critical point of the warping function $\omega$.

Therefore, we are lead to consider the more general anisotropic problem

$$
-\operatorname{div}_{g}\left(a \nabla_{g} u\right)+a h u=a u^{p}, u>0 \quad \text { in }(M, g)
$$

where $(M, g)$ is a $N$-dimensional compact Riemannian manifold, $p>1, h \in C^{1}(M)$ and $a \in$ $C^{2}(M)$ with $\min _{M} a>0$. We will assume that the anisotropic operator $-\operatorname{div}_{g}\left(a \nabla_{g} u\right)+a h u$ is coercive in $H^{1}(M)$. Our main result reads as follows.

Theorem 1.1. If $\frac{N}{N-2}<p<\frac{N+2}{N-2}$, then the problem (4) has a solution which is singular at a point $\xi_{0} \in M$.

As a consequence of the previous theorem and the above discussion, we deduce

Theorem 1.2. Assume that $(\mathcal{M}, \mathfrak{g})$ is a warped product $M \times_{\omega} K$. If $0<k<\frac{n-2}{2}$ then there exists a solution invariant with respect to $K$ of

$$
-\Delta_{\mathfrak{g}} u+R_{\mathfrak{g}} u=u^{\frac{n+2}{n-2}}, u>0, \text { in }(\mathcal{M}, \mathfrak{g})
$$

which is singular on $\left\{\xi_{0}\right\} \times K$, where $\xi_{0}$ is any point on $M$. Furthermore, if $\xi_{0}$ is a critical point of $\omega$ then the submanifold $\left\{\xi_{0}\right\} \times K$ is minimal in $\mathcal{M}$.

The proof of Theorem (1.1) follows the same strategy developed in [MP96]. In particular, we will replace the $N$-dimensional manifold $M$ by a bounded smooth domain $\Omega$ in $\mathbb{R}^{N}$ and we will focus on the Dirichlet boundary problem

$$
\begin{cases}-\operatorname{div}(a \nabla u)+a h u=a u^{p} & \text { in } \Omega \\ u=0 & \text { on } \partial \Omega \\ u>0 & \text { in } \Omega .\end{cases}
$$


Here $h \in C^{1}(M), a \in C^{2}(\bar{\Omega})$ with $\min _{\Omega} a>0$ and the anisotropic operator $-\operatorname{div}(a \nabla u)+a h u$ is coercive in $H_{0}^{1}(\Omega)$. We will show the following result

Theorem 1.3. If $\frac{N}{N-2}<p<\frac{N+2}{N-2}$, then the problem (5) has a solution which is singular at a point $\xi_{0} \in \Omega$.

The modification in the arguments to solve the problem on the manifold instead of in the domain are minor and are described in the last section of [MP96].

The paper is organized as follows. The proof of Theorem 1.3 is carried out in Section 3 and relies on the linear theory studied in Section 4 together with a contraction mapping argument developed in Section 5. All the necessary technical tools are contained in Section 2 and in the Appendix 6.

\section{Preliminaries}

2.1. Function spaces. For $\sigma>0$ we let $N_{\sigma}$ to be the ball $B_{\sigma}\left(\xi_{0}\right)$. For $\alpha \in(0,1), s \in(0, \sigma)$, $k \in \mathbb{N} \cup\{0\}$ and $\nu \in \mathbb{R}$ we define the seminorms

$$
|w|_{k, \alpha, s}:=\sum_{j=0}^{k} s^{j} \sup _{N_{s} \backslash N_{\frac{s}{2}}}\left|\nabla^{j} w\right|+s^{k+\alpha} \sup _{x, x^{\prime} \in N_{s} \backslash N_{\frac{s}{2}}} \frac{\left|\nabla^{k} w(x)-\nabla^{k} w\left(x^{\prime}\right)\right|}{\left|x-x^{\prime}\right|^{\alpha}},
$$

and the weighted Hölder norm $(\sigma>0$ is fixed)

$$
\|w\|_{C_{\nu}^{k, \alpha}}:=|w|_{C^{k, \alpha}\left(\bar{\Omega} \backslash N_{\frac{\sigma}{2}}\right)}+\sup _{0<s<\sigma} s^{-\nu}|w|_{k, \alpha, s} .
$$

The weighted Hölder space $C_{\nu}^{k, \alpha}(\Omega \backslash \Sigma)$ is defined by (here $\Sigma=\left\{\xi_{0}\right\}$ )

$$
C_{\nu}^{k, \alpha}(\Omega \backslash \Sigma):=\left\{w \in C_{l o c}^{k, \alpha}(\bar{\Omega} \backslash \Sigma):\|w\|_{C_{\nu}^{k, \alpha}}<\infty\right\} .
$$

The subspace of $C_{\nu}^{k, \alpha}(\Omega \backslash \Sigma)$ with Dirichlet boundary conditions will be denoted by

$$
C_{\nu, \mathcal{D}}^{k, \alpha}(\Omega \backslash \Sigma):=\left\{w \in C_{\nu}^{k, \alpha}(\Omega \backslash \Sigma): w=0 \text { on } \partial \Omega\right\} .
$$

The space $C_{\nu, \nu^{\prime}}^{k, \alpha}\left(\mathbb{R}^{N} \backslash\{0\}\right)$ is defined by

$$
\|w\|_{C_{\nu, \nu^{\prime}}^{k, \alpha}\left(\mathbb{R}^{N} \backslash\{0\}\right)}:=\|w\|_{C_{\nu}^{k, \alpha}\left(B_{2} \backslash\{0\}\right)}+\sup _{r \geq 1}\left(r^{-\nu^{\prime}}\|w(r \cdot)\|_{C^{k, \alpha}\left(\bar{B}_{2} \backslash B_{1}\right)}\right) .
$$

We now list some useful properties of the space $C_{\nu}^{k, \alpha}(\Omega \backslash \Sigma)$, see e.g. [MP96] and the book [PR00].

Lemma 2.1. The following properties hold.

i) If $w \in C_{\gamma}^{k+1, \alpha}(\Omega \backslash \Sigma)$ then $\nabla w \in C_{\gamma-1}^{k, \alpha}(\Omega \backslash \Sigma)$.

ii) If $w \in C_{\gamma}^{k+1,0}(\Omega \backslash \Sigma)$ then $w \in C_{\gamma}^{k, \alpha}(\Omega \backslash \Sigma)$ for every $\alpha \in[0,1)$.

iii) For every $w_{i} \in C_{\gamma_{i}}^{k, \alpha}(\Omega \backslash \Sigma)$, $i=1,2$, we have

$$
\left\|w_{1} w_{2}\right\|_{k, \gamma_{1}+\gamma_{2}, \alpha} \leq C\left\|w_{1}\right\|_{k, \gamma_{1}, \alpha}\left\|w_{2}\right\|_{k, \gamma_{2}, \alpha},
$$

for some $C>0$ independent of $w_{1}, w_{2}$.

iv) There exists $C>0$ such that for every $w \in C_{\gamma}^{k, \alpha}(\Omega \backslash \Sigma)$ with $w>0$ in $\bar{\Omega} \backslash \Sigma$ we have

$$
\left\|w^{p}\right\|_{k, \gamma, \alpha} \leq C\|w\|_{k, \gamma, \alpha}^{p} .
$$


2.2. The singular solution. The building block for our theory is the existence of a singular solution with different behaviour at the origin and at infinity. The following theorem provides such a solution.

Theorem 2.2 ([MP96]). Suppose that $\frac{N}{N-2}<p<\frac{N+2}{N-2}$. Then for every $\beta>0$ there exists a unique radial solution $u$ to

$$
\left\{\begin{array}{l}
-\Delta u=u^{p} \quad \text { in } \mathbb{R}^{N} \backslash\{0\} \\
u>0 \text { in } \mathbb{R}^{N} \backslash\{0\} \\
\lim _{|x| \rightarrow 0} u(x)=\infty
\end{array}\right.
$$

such that

$$
\lim _{r \rightarrow \infty} r^{N-2} u(r)=\beta, \quad \lim _{r \rightarrow 0^{+}} r^{\frac{2}{p-1}} u(r)=c_{p}:=[k(p, N)]^{\frac{1}{p-1}},
$$

where

$$
k(p, N)=\frac{2}{p-1}\left(N-\frac{2 p}{p-1}\right) .
$$

Let $u$ be a singular radial solution to (7). Then $u_{\varepsilon}(x):=\varepsilon^{-\frac{2}{p-1}} u\left(\frac{x}{\varepsilon}\right)$ is also a solution to (7). Note that

$$
u_{\varepsilon}(x) \leq C(\delta, u) \varepsilon^{N-2-\frac{2}{p-1}} \text { for }|x| \geq \delta,
$$

which shows that $u_{\varepsilon} \rightarrow 0$ locally uniformly in $\mathbb{R}^{N} \backslash\{0\}$. Due to this scaling and the asymptotic behavior of $u$ at infinity, for a given $\alpha>0$, we can find a solution $u_{1}$ such that

$$
r^{2} u_{1}^{p-1}(r) \leq \alpha \quad \text { on }(1, \infty) .
$$

2.3. The linearized operator around the singular solution. We consider the linearized operator

$$
L_{1}=\Delta+p u_{1}^{p-1}
$$

where in polar coordinates we denote

$$
\Delta=\frac{\partial^{2}}{\partial r^{2}}+\frac{N-1}{r} \frac{\partial}{\partial r}+\frac{1}{r^{2}} \Delta_{\theta} .
$$

Following [MP96], we recall that $\gamma_{j}$ is an indicial root of $L_{1}$ at 0 if $L_{1}\left(|x|^{\gamma_{j}} \varphi_{j}\right)=o\left(|x|^{\gamma-2}\right)$, where $\varphi_{j}$ is the $j$-th eigenfunction of $-\Delta_{\theta}$ on $S^{N-1}$, that is $-\Delta_{\theta} \varphi_{j}=\lambda_{j} \varphi_{j}$,

$$
\lambda_{0}=0, \quad \lambda_{j}=N-1, \quad \text { for } j=1, \ldots, N,
$$

and so on. Setting

$$
A_{p}:=p \lim _{r \rightarrow 0} r^{2} u_{1}^{p-1}(r)=p k(p, N)
$$

we have that

$$
\gamma_{j}^{ \pm}=\frac{1}{2}\left[2-N \pm \sqrt{(N-2)^{2}+4\left(\lambda_{j}-A_{p}\right)}\right] .
$$

For $\frac{N}{N-2}<p<\frac{N+2}{N-2}$ we have that ( $\Re$ denotes the real part)

$$
2-N<-\frac{2}{p-1}<\Re\left(\gamma_{0}^{-}\right) \leq \frac{2-N}{2} \leq \Re\left(\gamma_{0}^{+}\right)<0
$$

and

$$
\gamma_{j}^{-}<-\frac{2}{p-1} \text { for } j \geq 1 .
$$

Since $\lim _{r \rightarrow \infty} r^{2} u_{1}^{p-1}(r)=0$, the indicial roots of $L_{1}$ at infinity are the same as for the $\Delta$ itself. These values are given by

$$
\tilde{\gamma}_{j}^{ \pm}=\frac{1}{2}\left[2-N \pm \sqrt{(N-2)^{2}+4 \lambda_{j}}\right] .
$$


We shall choose $\mu, \nu$ in the region

$$
\frac{-2}{p-1}<\nu<\min \left\{\frac{-2}{p-1}+1, \Re\left(\gamma_{0}^{-}\right)\right\} \leq \frac{2-N}{2} \leq \Re\left(\gamma_{0}^{+}\right)<\mu<0,
$$

so that $\mu+\nu=2-N$.

We have the following propositions whose proofs can be found in [MP96].

Proposition 2.3. Let $w \in C_{\mu, 0}^{2, \alpha}\left(\mathbb{R}^{N} \backslash\{0\}\right)$ be a solution to $L_{1} w=0$. Then $w \equiv 0$.

Proposition 2.4. Let $w \in C_{\gamma, \gamma}^{2, \alpha}\left(\mathbb{R}^{N} \backslash\{0\}\right)$ be a solution to

$$
\Delta w+\frac{A_{p}}{r^{2}} w=0 \quad \text { in } \mathbb{R}^{N} \backslash\{0\},
$$

where $A_{p}$ is given by (8). If $\gamma$ is not an indicial root of the operator $\Delta+\frac{A_{p}}{r^{2}}$ then $w \equiv 0$.

\section{A SCHEME OF THE PROOF}

Let $\Sigma=\left\{\xi_{0}\right\} \subset \Omega$. To construct a solution to (5) which is singular precisely at the point $\xi_{0}$, we start by constructing an approximate solution to (5) which is singular exactly on $\Sigma$. For $\sigma>0$ small (to be chosen later) let us first fix a non-negative cut-off function $\chi \in C_{c}^{\infty}\left(B_{\sigma}\left(\xi_{0}\right)\right)$ such that $\chi=1$ in $B_{\frac{\sigma}{2}}\left(\xi_{0}\right)$. An approximate solution $\bar{u}_{\varepsilon}$ is defined by

$$
\bar{u}_{\varepsilon}(x)=\chi(x) u_{\varepsilon}\left(x-\xi_{0}\right)=\varepsilon^{-\frac{2}{p-1}} \chi(x) u_{1}\left(\frac{x-\xi_{0}}{\varepsilon}\right) .
$$

We shall look for positive solutions of the form $u=\bar{u}_{\varepsilon}+v$. Then, $v$ has to satisfy

$$
L_{\varepsilon} v+f_{\varepsilon}+Q(v)=0,
$$

where the linear operator $L_{\varepsilon}$ is

$$
L_{\varepsilon} v:=\operatorname{div}(a \nabla v)+a\left[p \bar{u}_{\varepsilon}^{p-1}-h\right] v,
$$

the error term is

$$
f_{\varepsilon}:=\operatorname{div}\left(a \nabla \bar{u}_{\varepsilon}\right)-a h \bar{u}_{\varepsilon}+a \bar{u}_{\varepsilon}^{p},
$$

and the non-linear term $Q$ is

$$
Q(v)=a\left[\left|\bar{u}_{\varepsilon}+v\right|^{p}-\bar{u}_{\varepsilon}^{p}-p \bar{u}_{\varepsilon}^{p-1} v\right] .
$$

To prove existence of solution to (10) we will use a fixed point argument on the space $C_{\nu, \mathcal{D}}^{2, \alpha}(\Omega \backslash \Sigma)$ for a suitable $\nu$. We note that if $v \in C_{\nu, \mathcal{D}}^{2, \alpha}(\Omega \backslash \Sigma)$ solves (10) then by maximum principle we have that $\bar{u}_{\varepsilon}+v>0$ in $\Omega$. This is a simple consequence of the fact that we will choose $\nu>-\frac{2}{p-1}$, and therefore, $\bar{u}_{\varepsilon}+v>0$ in a small neighborhood of $\Sigma$, thanks to the asymptotic behavior of $\bar{u}_{\varepsilon}$, around the origin and the coercivity assumption on $h$.

First of all, we estimate the size of the error term.

Lemma 3.1. The error $f_{\varepsilon}$ satisfies

$$
\left\|f_{\varepsilon}\right\|_{C_{\gamma-2}^{0, \alpha}} \leq C_{\gamma} \max \left\{\varepsilon^{1-\gamma-\frac{2}{p-1}}, \varepsilon^{N-\frac{2 p}{p-1}}\right\} \quad \text { for } \varepsilon>0 \text { small, }
$$

for every $\gamma<1-\frac{2}{p-1}$.

Proof. We only estimate the first term in (6), the estimate for the second term should having the same order as of the first one. It follows that

$$
u_{\varepsilon}\left(x-\xi_{0}\right) \approx \begin{cases}\left|x-\xi_{0}\right|^{-\frac{2}{p-1}} & \text { for }\left|x-\xi_{0}\right| \leq \varepsilon \\ \frac{\varepsilon^{N-\frac{2 p}{p-1}}}{\left|x-\xi_{0}\right|^{N-2}} & \text { for }\left|x-\xi_{0}\right| \geq \varepsilon,\end{cases}
$$


and

$$
\left|\nabla u_{\varepsilon}\left(x-\xi_{0}\right)\right| \leq C \begin{cases}\left|x-\xi_{0}\right|^{-\frac{2}{p-1}-1} & \text { for }\left|x-\xi_{0}\right| \leq \varepsilon \\ \frac{\varepsilon^{N-\frac{2 p}{p-1}}}{\left|x-\xi_{0}\right|^{N-1}} & \text { for }\left|x-\xi_{0}\right| \geq \varepsilon\end{cases}
$$

We write

$$
f_{\varepsilon}=a\left[\Delta \bar{u}_{\varepsilon}+\bar{u}_{\varepsilon}^{p}\right]+\nabla \bar{u}_{\varepsilon} \cdot \nabla a-a h \bar{u}_{\varepsilon} .
$$

Since $\chi \equiv 1$ in a small neighborhood of $\xi_{0}$, we have

$$
a\left|\Delta \bar{u}_{\varepsilon}+\bar{u}_{\varepsilon}^{p}\right| \leq C \varepsilon^{N-\frac{2 p}{p-1}} \quad \text { and } u_{\varepsilon}\left(x-\xi_{0}\right)|\nabla \chi \cdot \nabla a| \leq \varepsilon^{N-\frac{2 p}{p-1}} .
$$

Moreover,

$$
\begin{gathered}
\left|x-\xi_{0}\right|^{2-\gamma}\left|\nabla u_{\varepsilon}\left(x-\xi_{0}\right)\right||\nabla a(x)| \leq C \varepsilon^{1-\gamma-\frac{2}{p-1}}, \\
\left|x-\xi_{0}\right|^{2-\gamma} \bar{u}_{\varepsilon}\left(x-\xi_{0}\right) \leq C \varepsilon^{2-\gamma-\frac{2}{p-1}} .
\end{gathered}
$$

The lemma follows.

Next, we use the linear theory of $L_{\varepsilon}$ developed in the Section 4 and, applying the inverse of $L_{\varepsilon}$, that is $G_{\varepsilon}$, we rewrite the above equation (10) as

$$
v+G_{\varepsilon} f_{\varepsilon}+G_{\varepsilon} Q(v)=0 .
$$

The crucial fact we shall use is that the norm of $G_{\varepsilon}$ is uniformly bounded if $\varepsilon$ is sufficiently small.

By Lemma 3.1, the error $f_{\varepsilon}$ satisfies the estimate

$$
\left\|f_{\varepsilon}\right\|_{0, \alpha, \nu-2} \leq C \varepsilon^{q}, \quad q:=\min \left\{N-\frac{2 p}{p-1}, 1-\nu-\frac{2}{p-1}\right\} .
$$

Then, there exists $C_{0}>0$ such that $\left\|G_{\varepsilon} f_{\varepsilon}\right\|_{2, \alpha, \nu} \leq C_{0} \varepsilon^{q}$. This suggests to work on the ball

$$
\mathcal{B}_{\varepsilon, M}=\left\{v \in C_{\nu}^{2, \alpha}:\|v\|_{2, \alpha, \nu} \leq M \varepsilon^{q}\right\},
$$

for some $M>2 C_{0}$ large. In Section 5 we shall show that the map $v \mapsto G_{\varepsilon}\left[f_{\varepsilon}+Q(v)\right]$ is a contraction on the ball $\mathcal{B}_{\varepsilon, M}$ when $M$ is large and $\varepsilon$ is small enough. That will concludes our proof.

\section{The linear operator $L_{\varepsilon}$}

4.1. Injectivity of $L_{\varepsilon}$ on $C_{\mu, \mathcal{D}}^{2, \alpha}(\Omega \backslash \Sigma)$. In this section we study injectivity of the linearized operator

$$
L_{\varepsilon} w:=\operatorname{div}(a \nabla w)+a\left[p \bar{u}_{\varepsilon}^{p-1}-h\right] w .
$$

We shall use the following notations:

$$
\Omega_{\varepsilon}:=\Omega \backslash B_{\varepsilon}\left(\xi_{0}\right), \quad f^{+}:=\max \{f, 0\}, \quad f^{-}:=\min \{f, 0\} .
$$

Lemma 4.1. After a suitable normalization of $u_{1}$, the operator $L_{\varepsilon}$ satisfies maximum principle in $\Omega_{\varepsilon}$ for $\varepsilon>0$ small. More precisely, if $w \in H^{1}\left(\Omega_{\varepsilon}\right)$ satisfies

$$
\begin{cases}L_{\varepsilon} w \geq 0 & \text { in } \Omega_{\varepsilon} \\ w \leq 0 & \text { on } \partial \Omega_{\varepsilon}\end{cases}
$$

then $w \leq 0$ in $\Omega_{\varepsilon}$. 
Proof. The crucial fact we shall use is that the operator

$$
v \mapsto-\operatorname{div}(a \nabla v)+a h v
$$

is coercive, that is, for some $c_{0}>0$ we have

$$
\int_{\Omega} a\left[|\nabla v|^{2}+h v^{2}\right] d x \geq c_{0} \int_{\Omega}|\nabla v|^{2} d x \quad \text { for every } v \in H_{0}^{1}(\Omega) .
$$

Since $w \leq 0$ on the boundary $\partial \Omega_{\varepsilon}$, by extending $w^{+}$by 0 on $B_{\varepsilon}\left(\xi_{0}\right)$ we see that $w^{+} \in H_{0}^{1}(\Omega)$. Multiplying the inequality $L_{\varepsilon} w \geq 0$ by $w^{+}$, and then integrating by parts we obtain

$$
\int_{\Omega} a\left[\left|\nabla w^{+}\right|^{2}+h\left(w^{+}\right)^{2}-p \bar{u}_{\varepsilon}^{p-1}\left(w^{+}\right)^{2}\right] d x=0
$$

We also have

$$
\int_{\Omega} \frac{\left(w^{+}\right)^{2}}{\left|x-\xi_{0}\right|^{2}} d x \leq \frac{4}{(N-2)^{2}} \int_{\Omega}\left|\nabla w^{+}\right|^{2} d x .
$$

If we normalize $u_{1}$ in such a way that

$$
\operatorname{pa}_{\varepsilon}^{p-1} \leq c_{0} \frac{(N-2)^{2}}{8} \frac{1}{\left|x-\xi_{0}\right|^{2}} \quad \text { on } \Omega_{\varepsilon},
$$

then we have

$$
\int_{\Omega}\left|\nabla w^{+}\right|^{2} d x=0
$$

We conclude the lemma.

Remark 1. The above proof shows that $L_{\varepsilon}$ satisfies maximum principle in $B_{\sigma}\left(\xi_{0}\right) \backslash B_{\varepsilon}\left(\xi_{0}\right)$ for $\varepsilon>0$ small.

Lemma 4.2. Fix $\varepsilon_{0}>0$ such that $L_{\varepsilon}$ satisfies maximum principle on $\Omega_{\varepsilon}$. Let $2-N<\gamma<0$ be fixed. Let $w_{\varepsilon}$ be a solution to $L_{\varepsilon} w_{\varepsilon}=f_{\varepsilon}$ on $\Omega_{\varepsilon}$ for some $f_{\varepsilon} \in C_{\gamma-2}^{0, \alpha}\left(\Omega_{\varepsilon}\right)$, and $0<\varepsilon \leq \varepsilon_{0}$. Assume that $w_{\varepsilon}=0$ on $\partial \Omega$. Then there exists $C>0$ such that

$$
\left\|w_{\varepsilon}\right\|_{2, \alpha, \gamma} \leq C\left(\left\|f_{\varepsilon}\right\|_{0, \alpha, \gamma-2}+\varepsilon^{-\gamma}\left\|w_{\varepsilon}\right\|_{C^{0}\left(\partial B_{\varepsilon}\left(\xi_{0}\right)\right.}\right) .
$$

Proof. For $\phi(x):=\left|x-\xi_{0}\right|^{\gamma}$ we have

$$
\Delta \phi(x)=c_{N, \gamma}\left|x-\xi_{0}\right|^{\gamma-2}, \quad c_{N, \gamma}:=\gamma(N+\gamma-2)<0 .
$$

Since

for $\sigma>0$ small we have that

$$
\nabla a \cdot \nabla \phi-a h \phi=O\left(\left|x-\xi_{0}\right|^{\gamma-1}\right),
$$

$$
a \Delta \phi+\nabla a \cdot \nabla \phi-a h \phi \leq \frac{c_{N, \gamma}}{2} a\left|x-\xi_{0}\right|^{\gamma-2} \text { on } B_{\sigma}\left(\xi_{0}\right) .
$$

This shows that for a suitable choice of $u_{1}$, we have for some $\delta>0$

$$
L_{\varepsilon} \phi(x) \leq-\delta\left|x-\xi_{0}\right|^{\gamma-2} \quad \text { on } \boldsymbol{\Omega}:=B_{\sigma}\left(\xi_{0}\right) \backslash B_{\varepsilon}\left(\xi_{0}\right) .
$$

Therefore, we can choose $c_{1, \varepsilon} \approx\left\|f_{\varepsilon}\right\|_{0, \alpha, \gamma-2}$ so that

$$
L_{\varepsilon}\left(w_{\varepsilon}+c_{1, \varepsilon} \phi\right) \leq 0 \quad \text { on } \boldsymbol{\Omega} \text {. }
$$

We can also choose

$$
c_{2, \varepsilon} \approx \varepsilon^{-\gamma}\left\|w_{\varepsilon}\right\|_{C^{0}\left(\partial B_{\varepsilon}\left(\xi_{0}\right)\right)}+\left\|w_{\varepsilon}\right\|_{C^{0}\left(\partial B_{\sigma}\left(\xi_{0}\right)\right)}=: c_{3, \varepsilon}+c_{4, \varepsilon},
$$

so that

$$
w_{\varepsilon}+\left(c_{1, \varepsilon}+c_{2, \varepsilon}\right) \phi \geq 0 \quad \text { on } \partial \boldsymbol{\Omega} .
$$

Then by Maximum principle we have that (to get the other inequality use $-\phi$ )

$$
\left|w_{\varepsilon}\right| \leq\left(c_{1, \varepsilon}+c_{2, \varepsilon}\right) \phi \quad \text { in } \boldsymbol{\Omega} .
$$

Since, $L_{\varepsilon} w_{\varepsilon}=f_{\varepsilon}$ in $\Omega_{\varepsilon} \backslash \boldsymbol{\Omega}$, and $w_{\varepsilon}=0$ on $\partial \Omega$, we get that

$$
\left|w_{\varepsilon}(x)\right| \lesssim\left(c_{1, \varepsilon}+c_{2, \varepsilon}\right) \quad \text { for } x \in \Omega_{\varepsilon} \backslash \boldsymbol{\Omega} .
$$


We claim that

$$
c_{4, \varepsilon} \lesssim c_{3, \varepsilon}+\left\|f_{\varepsilon}\right\|_{0, \alpha, \gamma-2} .
$$

We assume by contradiction that the above claim is false. Then there exists a family of solutions $w_{\ell}=w_{\varepsilon_{\ell}}$ to $L_{\varepsilon_{\ell}} w_{\ell}=f_{\ell}$ with $0<\varepsilon_{\ell}<\varepsilon_{0}, f_{\ell} \in C_{\gamma-2}^{0, \alpha}\left(\Omega_{\varepsilon_{\ell}}\right), w_{\ell}=0$ on $\partial \Omega$ such that

$$
c_{4, \varepsilon_{\ell}}=1 \text { and } c_{3, \varepsilon_{\ell}}+\left\|f_{\ell}\right\|_{0, \alpha, \gamma-2} \rightarrow 0 .
$$

Then, up to a subsequence, $\Omega_{\varepsilon_{\ell}} \rightarrow \tilde{\Omega}_{\tilde{\varepsilon}}$, where

$$
\tilde{\Omega}_{\tilde{\varepsilon}}=\Omega \backslash\left\{\xi_{0}\right\} \quad \text { if } \tilde{\varepsilon}=0, \quad \text { and } \tilde{\Omega}_{\tilde{\varepsilon}}=\Omega_{\tilde{\varepsilon}}=\Omega \backslash B_{\tilde{\varepsilon}}\left(\xi_{0}\right) \text { if } \tilde{\varepsilon}>0 .
$$

From the estimates on $w_{\ell}$ we see that $w_{\ell} \rightarrow w$ in $\tilde{\Omega}_{\tilde{\varepsilon}}$. Moreover, $w$ satisfies

$$
\begin{cases}L_{\tilde{\varepsilon}} w=\operatorname{div}(a \nabla w)-a h w+p \bar{u}_{\tilde{\varepsilon}}^{p-1} w=0 & \text { in } \tilde{\Omega}_{\tilde{\varepsilon}} \\ w=0 & \text { on } \partial \Omega .\end{cases}
$$

Here, for $\tilde{\varepsilon}=0$ the function $\bar{u}_{\tilde{\varepsilon}}$ is considered to be identically zero.

If $\tilde{\varepsilon}>0$ then by Lemma 4.1 we get that $w \equiv 0$. Next we consider the case $\tilde{\varepsilon}=0$. We have that $w(x)=O\left(\left|x-\xi_{0}\right|^{\gamma}\right)$, and hence the singularity at $\xi_{0}$ is removable (note that $\gamma>2-N$ ), that is, $L_{\tilde{\varepsilon}} w=0$ weakly in $\Omega$. Thus, we can use coercivity hypothesis on $h$ to conclude that $w \equiv 0$. This contradicts the first condition in (15).

In this way we have that there exists $C>0$ independent of $\varepsilon$, but depending only on the right hand side of (14) such that

$$
\left|w_{\varepsilon}\right| \leq C \phi \quad \text { in } \Omega_{\varepsilon} .
$$

The desired estimate follows from Lemma 6.4 and a scaling argument (see e.g. [PR00, Chapter 2.2.1]).

Lemma 4.3. There exists $\varepsilon_{0}>0$ sufficiently small such that if $\varepsilon<\varepsilon_{0}$ then

$$
L_{\varepsilon}: C_{\mu, \mathcal{D}}^{2, \alpha}(\Omega \backslash \Sigma) \rightarrow C_{\mu-2}^{0, \alpha}(\Omega \backslash \Sigma)
$$

is injective.

Proof. We assume by contradiction that $L_{\varepsilon^{\ell}}$ is not injective for some $\varepsilon^{\ell} \rightarrow 0$. Let $w_{\ell} \in$ $C_{\mu, \mathcal{D}}^{2, \alpha}(\Omega \backslash \Sigma)$ be a non-trivial solution to $L_{\varepsilon^{\ell}} w_{\ell}=0$. We normalize $w_{\ell}$ so that

$$
\max _{\partial \Omega_{\varepsilon} \ell} \rho(x)^{-\mu}\left|w_{\ell}(x)\right|=\left(\varepsilon^{\ell}\right)^{-\mu} \max _{\partial \Omega_{\varepsilon} \ell}\left|w_{\ell}(x)\right|=1,
$$

where $\rho(x)=\left|x-\xi_{0}\right|$ in a small neighborhood of $\xi_{0}$, and outside it is a smooth positive function. Then by Lemma 4.2 we get that

$$
\sup _{\Omega_{\varepsilon \ell}}\left(\rho(x)^{-\mu}\left|w_{\ell}(x)\right|+\rho(x)^{-\mu+1}\left|\nabla w_{\ell}(x)\right|\right) \leq C .
$$

We set

$$
\tilde{w}_{\ell}(x)=\left(\varepsilon^{\ell}\right)^{-\mu} w_{\ell}\left(\varepsilon^{\ell} x+\xi_{0}\right), \quad|x|<R_{\ell}:=\frac{\sigma}{2 \varepsilon^{\ell}} .
$$

Then

where

$$
\Delta \tilde{w}_{\ell}(x)+p u_{1}^{p-1} \tilde{w}_{\ell}(x)=f_{\ell}(x),
$$

$$
\begin{aligned}
f_{\ell}(x) & :=\left(\varepsilon^{\ell}\right)^{2-\mu}\left(h w_{\ell}-a^{-1} \nabla a \cdot \nabla w_{\ell}\right), \quad y:=\varepsilon^{\ell} x+\xi_{0} \\
& =\left(\varepsilon^{\ell}\right)^{2} h(y) \tilde{w}_{\ell}(x)-\varepsilon^{\ell} a(y)^{-1} \nabla a(y) \cdot \nabla \tilde{w}_{\ell}(x) .
\end{aligned}
$$

It follows from (17) that $\tilde{w}_{\ell} \rightarrow \tilde{w}_{\infty}$ and $f_{\ell} \rightarrow 0$ in $C_{l o c}^{2}\left(\mathbb{R}^{N} \backslash B_{1}\right)$ and $C_{l o c}^{1}\left(\mathbb{R}^{N} \backslash B_{1}\right)$ respectively.

Next we show that $\tilde{w}_{\ell}$ is bounded in $C_{l o c}^{2}\left(B_{2} \backslash\{0\}\right)$. To this end it suffices to prove that

$$
S_{\ell}=\sup _{B_{2}}\left(|x|^{-\mu}\left|\tilde{w}_{\ell}(x)\right|+|x|^{-\mu+1}\left|\nabla \tilde{w}_{\ell}(x)\right|\right) \leq C .
$$


We assume by contradiction that the above supremum is not uniformly bounded. Let $0 \neq$ $x_{\ell} \in B_{2}$ be such that

$$
S_{\ell} \approx\left|x_{\ell}\right|^{-\mu}\left|\tilde{w}_{\ell}\left(x_{\ell}\right)\right|+\left|x_{\ell}\right|^{1-\mu}\left|\nabla \tilde{w}_{\ell}\left(x_{\ell}\right)\right| \text {. }
$$

We claim that $\left|x_{\ell}\right| \rightarrow 0$. On the contrary, if $x_{\ell} \rightarrow x_{\infty} \neq 0$, then setting $\bar{w}_{\ell}=\frac{w_{\ell}}{S_{\ell}}$ we see that $\bar{w}_{\ell} \rightarrow \bar{w}_{\infty}$, where

$$
L_{1} \bar{w}_{\infty}=0 \quad \text { in } B_{2} \backslash\{0\}, \quad \bar{w}_{\infty} \equiv 0 \text { in } B_{2} \backslash B_{1} .
$$

Therefore, $\bar{w}_{\infty} \equiv 0$ in $B_{2}$, which contradicts to

$$
\left|x_{\infty}\right|^{-\mu}\left|\bar{w}_{\infty}\left(x_{\infty}\right)\right|+\left|x_{\infty}\right|^{1-\mu}\left|\nabla \bar{w}_{\infty}\left(x_{\infty}\right)\right| \approx 1 .
$$

Thus we get that $x_{\ell} \rightarrow 0$.

Now we set

Then, for every $\delta>0$ and $\ell$ large we have

$$
v_{\ell}(x)=\frac{r_{\ell}^{-\mu} \tilde{w}_{\ell}\left(r_{\ell} x\right)}{S_{\ell}}, \quad r_{\ell}:=\left|x_{\ell}\right| .
$$

$$
L_{1} v_{\ell}=o_{\ell}(1), \quad|x|^{\mu}\left|v_{\ell}\right|+|x|^{1+\mu}\left|\nabla v_{\ell}\right| \leq C \quad \text { for } \delta \leq|x| \leq \frac{1}{\delta} .
$$

Therefore, up to a subsequence, $v_{\ell} \rightarrow v_{\infty}$ where $v_{\infty}$ satisfies

$$
\Delta v_{\infty}+\frac{p \kappa(p, N)}{|x|^{2}} v_{\infty}=0, \quad\left|v_{\infty}(x)\right| \leq C|x|^{\mu} \quad \text { in } \mathbb{R}^{N} \backslash\{0\} .
$$

Hence, by Proposition 2.4 we have $v_{\infty} \equiv 0$, a contradiction to $\max _{\partial B_{1}}\left(\left|v_{\infty}\right|+\left|\nabla v_{\infty}\right|\right) \approx 1$. This proves that $S_{\ell} \leq C$, and consequently we obtain that $\tilde{w}_{\ell} \rightarrow \tilde{w}_{\infty}$ in $C_{l o c}^{1}\left(\mathbb{R}^{N} \backslash\{0\}\right)$. Then the limit function $\tilde{w}_{\infty}$ would satisfy

$$
\Delta \tilde{w}_{\infty}+p u_{1}^{p-1} \tilde{w}_{\infty}=0 \quad \text { in } \mathbb{R}^{N} \backslash\{0\}, \quad \tilde{w}_{\infty} \in C_{\mu, \mu}^{2, \alpha}\left(\mathbb{R}^{N} \backslash\{0\}\right) .
$$

Then by Proposition 2.3 we have $\tilde{w}_{\infty} \equiv 0$, a contradiction to (16).

4.2. Uniform surjectivity of $L_{\varepsilon}$ on $C_{\mu, \mathcal{D}}^{2, \alpha}(\Omega \backslash \Sigma)$. Instead of using general theory of edge operators as developed in [Maz91], we shall use the notes of Pacard [Paca, Pacb] and PacardRivière [PR00] for edge operators with point singularity. Denoting $\rho(x):=\left|x-\xi_{0}\right|$, the weighted space $L_{\delta}^{2}(\Omega \backslash \Sigma)$ is defined by (we may also simply write $L_{\delta}^{2}$ or $L_{\delta}^{2}(\Omega)$ )

$$
L_{\delta}^{2}(\Omega \backslash \Sigma):=\left\{w \in L_{l o c}^{2}(\Omega \backslash \Sigma): \int_{\Omega} \rho^{-2-2 \delta}|w|^{2} d x<\infty\right\} .
$$

Let $L_{-\delta}^{2}(\Omega \backslash \Sigma)$ be the dual of $L_{\delta}^{2}(\Omega \backslash \Sigma)$ with respect to the pairing

$$
L_{\delta}^{2}(\Omega \backslash \Sigma) \times L_{-\delta}^{2}(\Omega \backslash \Sigma) \ni\left(w_{1}, w_{2}\right) \longrightarrow \int_{\Omega} w_{1} w_{2} \rho^{-2} d x .
$$

We note that the following embedding is continuous

$$
C_{\gamma}^{k, \alpha}(\Omega \backslash \Sigma) \hookrightarrow L_{\delta}^{2}(\Omega \backslash \Sigma) \quad \text { for } \delta<\gamma+\frac{N-2}{2} .
$$

Lemma 4.4. Let $w \in L_{\delta}^{2}$ be a solution to

$$
L_{\varepsilon} w=0 \quad \text { in } \Omega \backslash\left\{\xi_{0}\right\} .
$$

Then $w \in C_{\delta-\frac{N-2}{2}}^{2, \alpha}(\tilde{\Omega})$ for every $\tilde{\Omega} \Subset \Omega$.

Proof. For $x_{0} \in \Omega$ with $d\left(x_{0}, \partial \Omega\right) \geq\left|x_{0}-\xi_{0}\right|$ we set

$$
v(x)=w\left(x_{0}+R x\right), \quad R=\frac{1}{2}\left|x-\xi_{0}\right|, \quad|x| \leq 1 .
$$

Then using the elliptic regularity for $v$, namely,

$$
\|v\|_{C^{0}\left(B_{\frac{1}{2}}\right)} \leq C\|v\|_{L^{2}\left(B_{1}\right)},
$$


one obtains that $|w(x)| \leq C|x|^{\delta-\frac{n-2}{2}}$ for $x$ in a small neighborhood of $\xi_{0}$. In fact, by elliptic regularity, this estimate also holds on compact sets in $\Omega$. The lemma follows by a scaling argument and Schauder regularity.

The natural domain $D\left(L_{\varepsilon}\right)$ of the operator $L_{\varepsilon}$ is the set of functions $w \in L_{\delta}^{2}$ such that the distributional derivative $L_{\varepsilon} w$ is in $L_{\delta-2}^{2}$. More precisely, $w \in D\left(L_{\varepsilon}\right)$ if there exists $f \in L_{\delta-2}^{2}$ such that $w$ satisfies $L_{\varepsilon} w=f$ in the sense of distributions in $\Omega \backslash \Sigma$. However, in order to identify the adjoint of $L_{\varepsilon}$ in a natural way, one has to consider a smaller space including the boundary condition $w=0$ on $\partial \Omega$, which well-defined as a trace according to the next estimate.

Together with Lemma 6.3 and a rescaling argument (see e.g., [Paca, Proposition 1.2.1]) one can show that the following elliptic estimate holds: for $r_{0}>0$ with $B_{2 r_{0}}\left(\xi_{0}\right) \subset \Omega$

$$
\sum_{\ell=1}^{2}\left\|\nabla^{\ell} w\right\|_{L_{\delta-\ell}^{2}\left(B_{r_{0}}\left(\xi_{0}\right)\right)} \leq C\left(\|f\|_{L_{\delta-2}^{2}\left(B_{2 r_{0}}\left(\xi_{0}\right)\right)}+\|w\|_{L_{\delta}^{2}\left(B_{2 r_{0}}\left(\xi_{0}\right)\right)}\right) .
$$

In our next lemma we bound the weighted norm $\|w\|_{L_{\delta}^{2}}$ by $L^{2}$ norm of $w$ and the weighted norm $\|f\|_{L_{\delta-2}^{2}}$ for some values of $\delta$.

Lemma 4.5. Assume that $\delta-\frac{N-2}{2} \notin\left\{\Re \gamma_{j}^{ \pm}: j=0,1, \ldots\right\}$. Then there exists a compact set $K \subset \bar{\Omega} \backslash\left\{\xi_{0}\right\}$ and $r>0$ such that

$$
\|w\|_{L_{\delta}^{2}\left(B_{r}\left(\xi_{0}\right)\right)} \leq C(\varepsilon)\left(\|f\|_{L_{\delta-2}^{2}(\Omega)}+\|w\|_{L^{2}(K)}\right) .
$$

Proof. Let $R>0$ be such that $B_{4 R}\left(\xi_{0}\right) \subset \Omega$. Applying Lemma 6.2 on the ball $B_{R}\left(\xi_{0}\right)$ we get that

$$
\|u\|_{L_{\delta}^{2}\left(B_{R}\left(\xi_{0}\right)\right)} \leq C_{1}\left[\|h\|_{L_{\delta-2}^{2}\left(B_{R}\left(\xi_{0}\right)\right)}\|+\| \nabla a \cdot \nabla w\left\|_{L_{\delta-2}^{2}\left(B_{R}\left(\xi_{0}\right)\right)}+\right\| u \|_{L^{2}(K)}\right],
$$

for some $C_{1}>0$ and for some compact set $K \subset \bar{B}_{R}\left(\xi_{0}\right) \backslash\left\{\xi_{0}\right\}$. Since $\|\nabla a\| \in L^{\infty}(\Omega)$, it follows that

$$
\lim _{r \rightarrow 0} \frac{\|\nabla a \cdot \nabla w\|_{L_{\delta-2}^{2}\left(B_{r}\left(\xi_{0}\right)\right)}}{\|\nabla w\|_{L_{\delta-1}^{2}\left(B_{r}\left(\xi_{0}\right)\right)}}=0 .
$$

Therefore, for $r>0$ small enough, the weighted norm $\|\nabla a \cdot \nabla w\|_{L_{\delta-2}^{2}\left(B_{r}\left(\xi_{0}\right)\right)}$ can be absorbed one the left hand side, thanks to (18). On the region $B_{R}\left(\xi_{0}\right) \backslash B_{r}\left(\xi_{0}\right)$, the weighted norm $\|\nabla a \cdot \nabla w\|_{L_{\delta-2}^{2}}$ is equivalent to $\|\nabla a \cdot \nabla w\|_{L^{2}}$, and this can be controlled by

$$
\|w\|_{L^{2}\left(B_{2 R}\left(\xi_{0}\right) \backslash B_{\frac{r}{2}}\left(\xi_{0}\right)\right.}+\|f\|_{L^{2}\left(B_{2 R}\left(\xi_{0}\right) \backslash B_{\frac{r}{2}}\left(\xi_{0}\right)\right.} .
$$

We conclude the lemma.

As a consequence of (18)-(19) one can prove the following lemma (see e.g., Chapter 9, [Pacb]).

Lemma 4.6. The operator $L_{\varepsilon}: L_{\delta}^{2} \rightarrow L_{\delta-2}^{2}$ is Fredholm, provided $\delta-\frac{N-2}{2} \notin\left\{\Re \gamma_{j}^{ \pm}: j=\right.$ $0,1, \ldots\}$.

We shall fix $\delta>0$ slightly bigger than $\mu+\frac{N-2}{2}$, where $\mu$ is fixed according to (9). Thanks to the previous comment on the domain of $L_{\varepsilon}$, the adjoint of the operator

$$
L_{\varepsilon}: L_{-\delta}^{2} \rightarrow L_{-\delta-2}^{2}
$$

is given by

$$
L_{\delta+2}^{2} \rightarrow L_{\delta}^{2}, \quad w \mapsto \rho^{2} L_{\varepsilon}\left(w \rho^{-2}\right) .
$$

Then the adjoint operator (21) is injective, and $L_{\varepsilon}$ in (20) is surjective. Using the isomorphism

$$
\rho^{2 \delta}: L_{\tilde{\delta}}^{2} \rightarrow L_{2 \delta+\tilde{\delta}}^{2}, \quad w \mapsto \rho^{2 \delta} w,
$$


we identify the adjoint operator as

$$
L_{\varepsilon}^{*}: L_{-\delta+2}^{2} \rightarrow L_{-\delta}^{2}, \quad w \mapsto \rho^{2-2 \delta} L_{\varepsilon}\left(w \rho^{2 \delta-2}\right) .
$$

Now we consider the composition

$$
\mathcal{L}=L_{\varepsilon} \circ L_{\varepsilon}^{*}: L_{-\delta+2}^{2} \rightarrow L_{-\delta-2}^{2}, \quad w \mapsto L_{\varepsilon}\left[\rho^{2-2 \delta} L_{\varepsilon}\left(w \rho^{2 \delta-2}\right)\right] .
$$

Then $\mathcal{L}$ is an isomorphism, and hence there exists a two sided inverse

$$
\mathcal{G}_{\varepsilon}: L_{-\delta-2}^{2} \rightarrow L_{-\delta+2}^{2} \text {. }
$$

Consequently, the right inverse of $L_{\varepsilon}$ is given by $G_{\varepsilon}:=L_{\varepsilon}^{*} \mathcal{G}_{\varepsilon}$. It follows that

$$
G_{\varepsilon}: C_{\nu-2}^{0, \alpha}(\Omega \backslash \Sigma) \rightarrow C_{\nu, \mathcal{D}}^{2, \alpha}(\Omega \backslash \Sigma)
$$

is bounded.

Lemma 4.7. Let $\varepsilon_{0}>0$ be as in Lemma 4.3. Then for $0<\varepsilon<\varepsilon_{0}$ the system $L_{\varepsilon} w_{1}=0$, $w_{1}=L_{\varepsilon}^{*} w_{2}$ with $w_{1} \in C_{\nu, \mathcal{D}}^{2, \alpha}(\Omega \backslash \Sigma)$ and $w_{2} \in C_{\nu+2, \mathcal{D}}^{4, \alpha}(\Omega \backslash \Sigma)$ has only trivial solution.

Proof. We set $w=\rho^{2 \delta-2} w_{2}$. Then $L_{\varepsilon}\left[\rho^{2-2 \delta} L_{\varepsilon} w\right]=0$. Multiplying the equation by $w$ and then integrating by parts we get

$$
0=\int_{\Omega} \rho^{2-2 \delta}\left|L_{\varepsilon} w\right|^{2} d x
$$

Since $\nu+2 \delta>\mu$, we have $w \in C_{\nu+2 \delta}^{2, \alpha}(\Omega \backslash \Sigma) \subset C_{\mu}^{2, \alpha}(\Omega \backslash \Sigma)$. Then by Lemma 4.3 we get that $w=0$, equivalently $w_{1}=w_{2}=0$.

Lemma 4.8. There exists $\varepsilon_{0}>0$ small such that if $0<\varepsilon<\varepsilon_{0}$, then the sequence of solutions $\left(w_{1, \varepsilon^{\ell}}\right) \subset C_{\nu, \mathcal{D}}^{2, \alpha}(\Omega \backslash \Sigma) \cap L_{\varepsilon^{\ell}}^{*}\left[C_{\nu+2, \mathcal{D}}^{4, \alpha}(\Omega \backslash \Sigma)\right]$ to $L_{\varepsilon^{\ell}} w_{1, \varepsilon^{\ell}}=f_{\varepsilon^{\ell}}$ is uniformly bounded in $C_{\nu}^{2, \alpha}(\Omega \backslash \Sigma)$, provided $\left(f_{\varepsilon^{\ell}}\right)$ is uniformly bounded in $C_{\nu-2}^{0, \alpha}(\Omega \backslash \Sigma)$.

Proof. Assume by contradiction that the lemma is false. Then there exists a sequence $\varepsilon^{\ell} \rightarrow 0$ and $w_{1, \varepsilon^{\ell}} \in C_{\nu, \mathcal{D}}^{2, \alpha}(\Omega \backslash \Sigma) \cap L_{\varepsilon^{\ell}}^{*}\left[C_{\nu+4, \mathcal{D}}^{4, \alpha}(\Omega \backslash \Sigma)\right]$ with $L_{\varepsilon^{\ell}} w_{1, \varepsilon^{\ell}}=f_{\varepsilon^{\ell}}$ such that $\left\|f_{\varepsilon^{\ell}}\right\|_{C_{\nu-2, \mathcal{D}}^{0, \alpha}(\Omega \backslash \Sigma)} \leq$ $C$, and $\left(w_{1, \varepsilon^{\ell}}\right)$ is not bounded in $C_{\nu}^{2, \alpha}(\Omega \backslash \Sigma)$. By Lemma 4.2

$$
\left\|w_{1, \varepsilon^{\ell}}\right\|_{C_{\nu}^{2, \alpha}\left(\Omega_{\varepsilon} \ell\right)} \leq C+C \max _{\partial B_{\varepsilon^{\ell}}\left(\xi_{0}\right)}\left(\varepsilon^{\ell}\right)^{-\nu}\left(\left|w_{1, \varepsilon^{\ell}}\right|+\varepsilon^{\ell}\left|\nabla w_{1, \varepsilon^{\ell}}\right|\right)=: C+C S_{\varepsilon^{\ell}} .
$$

We distinguish the following two cases.

Case $1 S_{\varepsilon^{\ell}} \leq C$.

In this case we proceed as in the proof of Lemma 4.3. Let $x_{\ell} \in B_{\varepsilon^{\ell}}\left(\xi_{0}\right)$ be such that $\sup _{B_{\varepsilon^{\ell}}\left(\xi_{0}\right)}\left(\rho^{-\nu}\left|w_{1, \varepsilon^{\ell}}\right|+\rho^{-\nu+1}\left|\nabla w_{1, \varepsilon^{\ell}}\right|\right) \approx\left|x_{\ell}-\xi_{0}\right|^{-\nu}\left(\left|w_{1, \varepsilon^{\ell}}\left(x_{\ell}\right)\right|+\left|x_{\ell}-\xi_{0}\right|\left|\nabla w_{1, \varepsilon^{\ell}}\left(x_{\ell}\right)\right|\right)=: S_{\ell} \rightarrow \infty$.

Then necessarily $r_{\ell}:=\left|x_{\ell}-\xi_{0}\right|=o\left(\varepsilon^{\ell}\right)$. Setting

$$
\tilde{w}_{1, \varepsilon^{\ell}}(x):=\frac{r_{\ell}^{-\nu} w_{1, \varepsilon^{\ell}}\left(r_{\ell} x+\xi_{0}\right)}{S_{\ell}}
$$

one would get that $\tilde{w}_{1, \varepsilon^{\ell}} \rightarrow \tilde{w}_{1} \not \equiv 0$ where

$$
\tilde{L}_{1} \tilde{w}_{1}=0 \quad \text { in } \mathbb{R}^{n} \backslash\{0\}, \quad r^{-\nu}\left|\tilde{w}_{1}\right| \leq C, \quad \tilde{L}_{1}:=\Delta+\frac{A_{p}}{r^{2}},
$$

where $A_{p}$ is as in (8). Since $\nu$ does not coincide with indicial roots of $\tilde{L}_{1}$, from Proposition 2.4 we get that $\tilde{w}_{1} \equiv 0$, a contradiction.

Case $2 S_{\varepsilon^{\ell}} \rightarrow \infty$. 
In this case we set

$$
\tilde{w}_{1, \varepsilon^{\ell}}(x)=\left(\varepsilon^{\ell}\right)^{-\nu} \frac{w_{1, \varepsilon^{\ell}}\left(\varepsilon^{\ell} x+\xi_{0}\right)}{S_{\varepsilon^{\ell}}} .
$$

Then $\max _{\partial B_{1}}\left(\left|\tilde{w}_{1, \varepsilon^{\ell}}\right|+\left|\nabla \tilde{w}_{1, \varepsilon^{\ell}}\right|\right) \approx 1$. Moreover, proceeding as before (see Lemma 4.3) we would get that $w_{1, \varepsilon^{\ell}} \rightarrow \tilde{w}_{1} \neq \equiv 0$ where

$$
L_{1} \tilde{w}_{1}=0 \quad \text { in } \mathbb{R}^{n} \backslash\{0\}, \quad r^{-\nu}\left|\tilde{w}_{1}\right| \leq C .
$$

Since $\tilde{w}_{1}$ decays at infinity, its decay rate is determined by the indicial roots of $L_{1}$ (which are exactly the same as $\Delta$ ) at infinity. In fact, $\tilde{w}_{1}$ would be bounded by $r^{2-N}$ at infinity, see e.g., [MP96].

Since $w_{1, \varepsilon^{\ell}} \in L_{\varepsilon^{\ell}}^{*}\left[C_{\nu+2, \mathcal{D}}^{4, \alpha}(\Omega \backslash \Sigma)\right]$, we have $w_{1, \varepsilon^{\ell}}=\rho^{2-2 \delta} L_{\varepsilon^{\ell}} w_{2, \varepsilon^{\ell}}$ for some $w_{2, \varepsilon^{\ell}} \in C_{\nu+2 \delta, \mathcal{D}}^{4, \alpha}(\Omega \backslash$ $\Sigma)$. Now we set

$$
\tilde{w}_{2, \varepsilon^{\ell}}(x):=\frac{\left(\varepsilon^{\ell}\right)^{-\nu-2 \delta} w_{2, \varepsilon^{\ell}}\left(\varepsilon^{\ell} x+\xi_{0}\right)}{S_{\varepsilon^{\ell}}} .
$$

Using that $2 \delta+\nu>\mu$, and following the proof of Lemma 4.3, one can show that the family $\tilde{w}_{2, \varepsilon^{\ell}}$ converges to a limit function $\tilde{w}_{2}$, where

$$
L_{1} \tilde{w}_{2}=|x|^{2 \delta-2} \tilde{w}_{1} \quad \text { in } \mathbb{R}^{n} \backslash\{0\}, \quad|x|^{-\nu-2 \delta}\left|\tilde{w}_{2}\right| \leq C .
$$

Thus, $L_{1}\left[r^{2-2 \delta} L_{1} \tilde{w}_{2}\right]=0$. We multiply this equation by $\tilde{w}_{2}$ and integrate it on $\mathbb{R}^{N}$. Then an integration by parts leads $L_{1} \tilde{w}_{2}=0$ (this is justified because of the decay of $\tilde{w}_{1}$ at infinity, provided we choose $\delta>0$ sufficiently close to $\mu+\frac{N-2}{2}$ ). Again, as $2 \delta+\nu>\mu$, by Proposition 2.3 we have $\tilde{w}_{2}=\tilde{w}_{1}=0$, a contradiction.

\section{The NON-LINEAR TERM $Q$}

Lemma 5.1. Let $M_{1}>1$ be fixed. Then for $\varepsilon_{0}<<1$ we have

$$
\left\|Q\left(v_{1}\right)-Q\left(v_{2}\right)\right\|_{0, \alpha, \nu-2} \leq \frac{1}{M_{1}}\left\|v_{1}-v_{2}\right\|_{2, \alpha, \nu}
$$

for every $v_{1}, v_{2} \in \mathcal{B}_{\varepsilon, M}:=\left\{v \in C_{\nu}^{2, \alpha}:\|v\|_{2, \alpha, \nu} \leq M \varepsilon^{q}\right\}$.

Proof. In Lemma 3.1, the error term $f_{\varepsilon}$ is bounded by the maximum of two terms. If the maximum is the second term $\varepsilon^{N-2 p /(p-1)}$, we argue as in [MP96]. Let us consider the case when the maximum is the first term. Let

$$
q_{1}:=\left(N-\frac{2 p}{p-1}\right)-\left(1-\nu-\frac{2}{p-1}\right)=N+\nu-3>0 .
$$

We start by showing that there exists $\tau>0$ small (independent of $\varepsilon<<1$ ) such that

$$
|v(x)| \leq \frac{1}{10} \bar{u}_{\varepsilon}(x) \quad \text { for every } x \in B_{\tau_{\varepsilon}}\left(\xi_{0}\right), v \in \mathcal{B}_{\varepsilon, M},
$$

where

$$
\tau_{\varepsilon}:=\tau \varepsilon^{\frac{q_{1}}{\nu-2+N}} \rightarrow 0 .
$$

To prove this we recall that there exists $c_{1}, c_{2}>1$ such that

$$
\begin{gathered}
\frac{1}{c_{1}} \leq|x|^{\frac{2}{p-1}} u_{\varepsilon}(x) \leq c_{1} \quad \text { for }|x| \leq \varepsilon \\
\frac{1}{c_{2}} \leq \varepsilon^{-N+\frac{2 p}{p-1}|x|^{N-2} u_{\varepsilon}(x) \leq c_{2} \quad \text { for } \varepsilon \leq|x| \leq \tau .}
\end{gathered}
$$

On the other hand,

$$
\varepsilon^{-N+\frac{2 p}{p-1}} \rho(x)^{-\nu}|v(x)| \leq M .
$$

As $\nu>\frac{-2}{p-1}$, we have $(22)$ for some $\tau>0$ small. 
We have

$$
\begin{aligned}
Q\left(v_{1}\right)-Q\left(v_{2}\right) & =a \int_{0}^{1} \frac{d}{d t}\left|\bar{u}_{\varepsilon}+v_{1}+t\left(v_{1}-v_{2}\right)\right|^{p} d t-p \bar{u}_{\varepsilon}^{p-1}\left(v_{1}-v_{2}\right) \\
& =a p\left(v_{1}-v_{2}\right) \int_{0}^{1}\left(\left|\bar{u}_{\varepsilon}+v_{1}+t\left(v_{1}-v_{2}\right)\right|^{p-1}-\bar{u}_{\varepsilon}^{p-1}\right) d t \\
& =: a p\left(v_{1}-v_{2}\right) \int_{0}^{1} Q\left(v_{1}, v_{2}\right) d t
\end{aligned}
$$

Next, using that

$$
(1+r)^{p-1}=1+O(|r|) \quad \text { for }|r| \leq \frac{1}{2},
$$

we estimate for $x \in B_{\varepsilon}\left(\xi_{0}\right)$

$$
\begin{aligned}
\left|Q\left(v_{1}, v_{2}\right)\right|(x) & \leq C \bar{u}_{\varepsilon}(x)^{p-1} \frac{\left|v_{1}\right|(x)+\left|v_{2}\right|(x)}{\bar{u}_{\varepsilon}(x)} \\
& \leq C M \varepsilon^{\frac{2}{p-1}+\nu+q} \rho^{-2}(x) \\
& =C M \varepsilon \rho(x)^{-2},
\end{aligned}
$$

and for $x \in B_{\tau_{\varepsilon}}\left(\xi_{0}\right) \backslash B_{\varepsilon}\left(\xi_{0}\right)$

$$
\begin{aligned}
\left|Q\left(v_{1}, v_{2}\right)\right|(x) & \leq C M \rho(x)^{-2} \max \left\{\varepsilon, \varepsilon^{\left(N-\frac{2 p}{p-1}\right)(p-2)+q} \tau_{\varepsilon}^{(2-N)(p-2)+\nu+2}\right\} \\
& =C M \rho(x)^{-2} o_{\varepsilon}(1) .
\end{aligned}
$$

Here we have used that the second term in the maximum is of the order $\varepsilon^{r}$ for some $r>0$. Indeed, from the definition of $\tau_{\varepsilon}, q$ and $q_{1}$, the exponent of $\varepsilon$ is

$$
\begin{aligned}
& \left(N-\frac{2 p}{p-1}\right)(p-2)+q+[(2-N)(p-2)+\nu+2]\left(1-\frac{1}{N+\nu-2}\right) \\
& =1-\frac{(2-N)(p-2)+\nu+2}{N+\nu-2} \\
& =\frac{(N-2)(p-1)-2}{N+\nu-2} \\
& >0
\end{aligned}
$$

where the last inequality follows from $p>\frac{N}{N-2}$ and $N+\nu-2>0$. Finally, as $\nu>-\frac{2}{p-1}$, we easily obtain for $x \in \Omega \backslash B_{\tau_{\varepsilon}}\left(\xi_{0}\right)$

$$
\begin{aligned}
\left|Q\left(v_{1}, v_{2}\right)\right|(x) & \leq C\left(\bar{u}_{\varepsilon}^{p-1}+\left|v_{1}\right|^{p-1}+\left|v_{2}\right|^{p-1}\right)(x) \\
& \leq C \rho(x)^{-2}\left(\bar{u}_{\varepsilon}^{p-1}(x) \rho(x)^{2}+M \varepsilon^{q(p-1)}\right) \\
& =o_{\varepsilon}(1) \rho(x)^{-2} .
\end{aligned}
$$

Combining these estimates we get for $\varepsilon<<1$

$$
\left\|Q\left(v_{1}\right)-Q\left(v_{2}\right)\right\|_{0,0, \nu-2}=o_{\varepsilon}(1)\left\|v_{1}-v_{2}\right\|_{0,0, \nu}=o_{\varepsilon}(1)\left\|v_{1}-v_{2}\right\|_{2, \alpha, \nu} .
$$

Next we estimate the weighted Hölder norm of $Q\left(v_{1}\right)-Q\left(v_{2}\right)$ with Hölder exponent $\alpha \leq$ $p-1$. For $0<s<\sigma$ we write

$$
\begin{aligned}
& s^{2-\nu+\alpha} \sup _{x, x^{\prime} \in N_{s} \backslash N_{\frac{s}{2}}} \frac{\left|\left[Q\left(v_{1}\right)-Q\left(v_{2}\right)\right](x)-\left[Q\left(v_{1}\right)-Q\left(v_{2}\right)\right]\left(x^{\prime}\right)\right|}{\left|x-x^{\prime}\right|^{\alpha}} \\
& \leq 4\left\|Q\left(v_{1}\right)-Q\left(v_{2}\right)\right\|_{0,0, \nu-2} \\
& +s^{2-\nu+\alpha} \sup _{x, x^{\prime} \in N_{s} \backslash N_{\frac{s}{2}},\left|x-x^{\prime}\right| \leq \frac{s}{4}} \frac{\left|\left[Q\left(v_{1}\right)-Q\left(v_{2}\right)\right](x)-\left[Q\left(v_{1}\right)-Q\left(v_{2}\right)\right]\left(x^{\prime}\right)\right|}{\left|x-x^{\prime}\right|^{\alpha}} .
\end{aligned}
$$


Notice that for $x, x^{\prime} \in N_{s} \backslash N_{\frac{s}{2}}$ with $\left|x-x^{\prime}\right| \leq \frac{s}{4}$, the line segment $[x, y]$ joining $x$ and $y$ lies in $N_{2 s} \backslash N_{\frac{s}{4}}$. The desired estimate follows on the ball $B_{\tau_{\varepsilon}}\left(\xi_{0}\right)$ by estimating $Q\left(v_{1}, v_{2}\right)(x)-$ $Q\left(v_{1}, v_{2}\right)\left(x^{\prime}\right)$ using the following gradient bound (we are using that $\left|\bar{u}_{\varepsilon}+v\right|^{p-1}$ is $C^{1}$ in this region)

$$
\begin{aligned}
\nabla Q\left(v_{1}, v_{2}\right)= & (p-1)\left[\left(\bar{u}_{\varepsilon}+v_{1}+t\left(v_{1}-v_{2}\right)^{p-2}-\bar{u}_{\varepsilon}^{p-2}\right)\right] \nabla \bar{u}_{\varepsilon} \\
& +(p-1)\left(\bar{u}_{\varepsilon}+v_{1}+t\left(v_{1}-v_{2}\right)^{p-2} \nabla\left[v_{1}+t\left(v_{1}-v_{2}\right)\right]\right. \\
= & O(1) \bar{u}_{\varepsilon}^{p-3}\left(\left|v_{1}\right|+\left|v_{2}\right|\right)\left|\nabla u_{\varepsilon}\right|+O(1) \bar{u}_{\varepsilon}^{p-2}\left(\left|\nabla v_{1}\right|+\left|\nabla v_{2}\right|\right) .
\end{aligned}
$$

In fact, gradient bounds can also be used for the region $B_{\tau_{\varepsilon}}\left(\xi_{0}\right)$ if $p \geq 2$. For $1<p \leq 2$, one can use the following inequalty

$$
\left\|\left.\phi\right|^{p-1}(x)-|\phi|^{p-1}\left(x^{\prime}\right)|\leq| \phi(x)-\left.\phi\left(x^{\prime}\right)\right|^{p-1} \leq\right\| \nabla \phi \|_{C^{0}\left(\left[x, x^{\prime}\right]\right)}^{p-1}\left|x-x^{\prime}\right|^{p-1},
$$

with $\phi=\bar{u}_{\varepsilon}$ and $\phi=\bar{u}_{\varepsilon}+v_{1}+t\left(v_{1}-v_{2}\right)$.

We conclude the lemma.

\section{Appendix}

The following lemma can be proven in the spirit of [Paca, Proposition 1.5.1]

Lemma 6.1. For $d \in \mathbb{R}$ set

$$
\delta_{j}:=\Re\left(\left(\frac{N-2}{2}\right)^{2}+\lambda_{j}-d\right)^{\frac{1}{2}}, \quad j \in \mathbb{N} .
$$

Then for $\delta \in \mathbb{R} \backslash\left\{ \pm \delta_{j}: j=0,1, \ldots\right\}$ there exists $C=C(N, \delta)$ such that if $u$ is a solution to

$$
\Delta u+\frac{d}{|x|^{2}} u=f \quad \text { in } B_{1} \backslash\{0\},
$$

then

$$
\|u\|_{L_{\delta}^{2}\left(B_{1}\right)} \leq C\left(\|f\|_{L_{\delta-2}^{2}\left(B_{1}\right)}+\|u\|_{L^{2}\left(B_{1} \backslash B_{\frac{1}{2}}\right)}\right) .
$$

Lemma 6.2. Let $\zeta$ be a continuous function in $\bar{B}_{1}$. Let $\delta_{j}$ be given by $(23)$ with $d=\zeta(0)$. Then for $\delta \in \mathbb{R} \backslash\left\{ \pm \delta_{j}: j=0,1, \ldots\right\}$ there exists a compact set $K \subset \bar{B}_{1} \backslash\{0\}$ and a constant $C>0$ such that for every $u \in L_{\delta}^{2}\left(B_{1}\right)$ solving

we have

$$
\Delta u+\frac{\zeta}{|x|^{2}} u=f \quad \text { in } B_{1} \backslash\{0\}, \quad f \in L_{\delta}^{2}\left(B_{1}\right)
$$

$$
\|u\|_{L_{\delta}^{2}\left(B_{1}\right)} \leq C\left(\|f\|_{L_{\delta-2}^{2}\left(B_{1}\right)}+\|u\|_{L^{2}(K)}\right) .
$$

Proof. We rewrite the equation as

$$
\Delta u+\frac{\zeta(0)}{|x|^{2}} u=f+\tilde{f}, \quad \tilde{f}:=\frac{\zeta(0)-\zeta}{|x|^{2}} u
$$

Then by Lemma 6.1 we get

$$
\|u\|_{L_{\delta}^{2}\left(B_{1}\right)} \leq C_{1}\left(\|f\|_{L_{\delta-2}^{2}\left(B_{1}\right)}+\|\tilde{f}\|_{L_{\delta-2}^{2}\left(B_{1}\right)}+\|u\|_{L^{2}\left(B_{1} \backslash B_{\frac{1}{2}}\right)}\right) .
$$

Let $r>0$ be sufficiently small so that $|\zeta-\zeta(0)| \leq \frac{1}{2 C_{1}}$ on $B_{r}$. Then

$$
\|\tilde{f}\|_{L_{\delta-2}^{2}\left(B_{1}\right)} \leq \frac{1}{2 C_{1}}\|u\|_{L_{\delta}^{2}\left(B_{r}\right)}+C\left(r,\|\zeta\|_{L^{\infty}\left(B_{1}\right)}\right)\|u\|_{L^{2}\left(B_{1} \backslash B_{r}\right)} .
$$

The proof follows by absorbing the term $\|u\|_{L_{\delta}^{2}\left(B_{r}\right)}$ on the left hand side, and taking $K=$ $\bar{B}_{1} \backslash B_{r}$. 
Lemma 6.3 ( $L^{2}$ estimate). Let $\Omega$ be a bounded open set in $\mathbb{R}^{n}$. Let $b_{i} \in L^{\infty}(\Omega)$ with

$$
\left\|b_{i}\right\|_{L^{\infty}(\Omega)} \leq \Lambda, \quad i=0,1, \ldots, n .
$$

Let $u \in L^{2}(\Omega)$ be a weak solution solution to

$$
\Delta u+\sum_{i=1}^{n} b_{i} \frac{\partial u}{\partial x_{i}}+b_{0} u=f \quad \text { in } \Omega,
$$

for some $f \in L^{2}(\Omega)$. Then for every $\tilde{\Omega} \Subset \Omega$ there exists $C=C(\tilde{\Omega}, \Lambda)$ such that

$$
\|u\|_{W^{2,2}(\tilde{\Omega})} \leq C\left(\|f\|_{L^{2}(\Omega)}+\|u\|_{L^{2}(\Omega)}\right) .
$$

Lemma 6.4 (Schauder estimate). Let $\Omega$ be a bounded open set in $\mathbb{R}^{n}$. Let $b_{i} \in C^{0, \alpha}(\Omega)$ with

$$
\left\|b_{i}\right\|_{C^{0, \alpha}(\Omega)} \leq \Lambda, \quad i=0,1, \ldots, n .
$$

Let $u$ be a classical solution to

$$
\Delta u+\sum_{i=1}^{n} b_{i} \frac{\partial u}{\partial x_{i}}+b_{0} u=f \quad \text { in } \Omega,
$$

for some $f \in C^{0, \alpha}(\Omega)$. Then for every $\tilde{\Omega} \Subset \Omega$ there exists $C=C(\tilde{\Omega}, \Lambda)$ such that

$$
\|u\|_{C^{2, \alpha}(\tilde{\Omega})} \leq C\left(\|f\|_{C^{0, \alpha}(\Omega)}+\|u\|_{C^{0}(\Omega)}\right) .
$$

Additionally, if $\Omega$ is regular, $\partial \Omega$ has two components $\Gamma_{1}$ and $\Gamma_{2}$, and if $u=0$ on $\Gamma_{1}$ then for $\tilde{\Omega} \Subset\left(\Omega \cup \Gamma_{1}\right)$ there exists $C=C(\tilde{\Omega}, \Lambda)$ such that

$$
\|u\|_{C^{2, \alpha}(\tilde{\Omega})} \leq C\left(\|f\|_{C^{0, \alpha}(\Omega)}+\|u\|_{C^{0}(\Omega)}\right) .
$$

\section{REFERENCES}

[CP16] Mónica Clapp and Angela Pistoia. Symmetries, Hopf fibrations and supercritical elliptic problems. In Mathematical Congress of the Americas, volume 656 of Contemp. Math., pages 1-12. Amer. Math. Soc., Providence, RI, 2016.

[Maz91] Rafe Mazzeo. Elliptic theory of differential edge operators. I. Comm. Partial Differential Equations, 16(10):1615-1664, 1991.

[MP96] Rafe Mazzeo and Frank Pacard. A construction of singular solutions for a semilinear elliptic equation using asymptotic analysis. J. Differential Geom., 44(2):331-370, 1996.

[MP99] Rafe Mazzeo and Frank Pacard. Constant scalar curvature metrics with isolated singularities. Duke Math. J., 99(3):353-418, 1999.

[MS91] Rafe Mazzeo and Nathan Smale. Conformally flat metrics of constant positive scalar curvature on subdomains of the sphere. J. Differential Geom., 34(3):581-621, 1991.

[Paca] F. Pacard. Analysis in weighted spaces. https://cel.archives-ouvertes.fr/cel-00392164/file/Pacard.pdf.

$[\mathrm{Pacb}]$ F. Pacard. Connected sum constructions in geometry and in nonlinear analysis. http://www.cmls.polytechnique.fr/perso/pacard/Publications/Lecture-Part-I.pdf.

[PR00] Frank Pacard and Tristan Rivière. Linear and nonlinear aspects of vortices, volume 39 of Progress in Nonlinear Differential Equations and their Applications. Birkhäuser Boston, Inc., Boston, MA, 2000. The Ginzburg-Landau model.

[Sch88] Richard M. Schoen. The existence of weak solutions with prescribed singular behavior for a conformally invariant scalar equation. Comm. Pure Appl. Math., 41(3):317-392, 1988.

(Ali Hyder) Department of Mathematics, Johns Hopkins University, 3400 N. Charles Street, BALTimoRE, MD 21218

E-mail address: ahyder4@jhu.edu

(Angela Pistoia) Dipartimento SBAi, "Sapienza" Università di Roma, via Antonio Scarpa 16, 00161 Roma, Italy

E-mail address: angela.pistoia@uniroma1.it

(Yannick Sire) Department of Mathematics, Johns Hopkins University, 3400 N. Charles Street, BALTimore, MD 21218

E-mail address: sire@jhu.edu 